\title{
Correlations between indirect and direct measurements of body composition
}

\section{By A. J. Kempster, Meat and Livestock Commission, PO Box 44, Queenszoay House, Bletchley, Milton Keynes MK2 $2 E F$}

Meat production is dominated by two factors. First there are the biological and agricultural considerations of resource use, in particular the efficiency with which feed is converted into meat. Second, there are marketing considerations, essentially the ability of producers to adapt output to conform more closely with changing market demand. The development of cost-effective methods for estimating body composition is an important prerequisite to improvements in both of these factors.

The value of a carcass depends on a number of different characteristics: (1) weight; (2) proportions of muscle, fat and bone; (3) distribution of the tissues through the carcass; (4) muscularity; (5) meat quality; (6) chemical composition.

The weight of the carcass has a major influence not only on the quantity of the various tissues, but also on the size of the muscles exposed on cutting which affects the butcher's ability to provide joints of suitable size for customer requirements. Among carcasses of similar weight, the proportion of muscle (hereafter referred to as lean) they contain is of major importance since this is a prime determinant of meat yield and commercial value to the butcher. Concern about the possible association between diet and health and calls to reduce fat consumption have recently reinforced the importance of increased lean:fat in meat (Committee on Medical Aspects of Food Policy, 1984).

Muscle weight distribution is potentially important because there are large differences in retail value between muscles from different parts of the carcass, but there is little variation to exploit commercially. More genetic and environmental variation exists in the way fat is partitioned between the subcutaneous, intermuscular, intramuscular and intra-abdominal depots (Kempster, 1980), and carcass-evaluation research is beginning to focus on methods of quantifying each depot separately or the relative size of depots. This is especially important in the case of intramuscular fat because there is concern that this depot should be maintained at levels adequate to ensure good eating quality while fat in other depots continues to be reduced. Accurate information on intramuscular fat content is also important in the diet and health debate because this sets the baseline fat consumption from meat for people who trim all visible fat.

The importance of differences in muscularity (independent of carcass lean content per se) is less obvious. Fresh-meat traders tend to prefer blockier carcasses with thicker muscles because the appearance of the joints prepared from them is sometimes better. There may also be advantages in terms of increased tenderness and reduced weight loss in the preparation and cooking, but the extent and 
significance of these advantages are not clear, and I shall not develop them further. Meat quality is also considered to be outside the scope of the present paper.

\section{The direct measurement of body composition: establishing the baseline}

The direct measurement of body composition to establish baseline characteristics is a necessity in any prediction application. Setting aside carcass weight, the description of carcass lean content is of most importance for the reasons outlined previously. We have argued, therefore, that the most satisfactory approach is the division of the carcass into standardized joints (either commercial joints or anatomical joints depending on the application) and their separation into component tissues (Kempster et al. 1982). Such tissue separation is now well established as baseline technique in most European countries and efforts are being made to standardize the methods, in particular to improve the accuracy of application of EEC carcass classification schemes in different countries. Baseline techniques in North America have generally been based on commercial boning and fat trimming, but a working party set up by the American Meat Science Association recommended that tissue-separation procedures similar to those used in Europe should now be used (Cross, 1983). Tissue-separation techniques can be supported by chemical analysis where this is necessary, for example, in nutritional studies or as a check on the standard of physical separation, but whole-body chemical analysis can be expensive and, without initial tissue separation, difficult to relate to commercial carcass valuation.

\section{Indirect measurement}

Three principal criteria are involved in the selection of the most appropriate indirect technique for a particular application: (I) the precision with which the technique is expected to predict baseline carcass characteristics (given as the correlation coefficient or now, more commonly, as the residual standard deviation, which is preferable because it is less sensitive to variation in the dependent variable); (2) the cost, including the practicability of taking the predicting measurements; (3) the stability of prediction equations to differences in measurement circumstances or the types of animals measured. King (1981) adds public acceptability to his list of criteria, giving as examples the human health risk associated with the use of radioactive isotopes and the unacceptability of biopsy techniques on welfare grounds. But these could be considered within the broad definition of practability.

In general terms, the best measurements will be those which give maximum precision in relation to cost. There is an extensive literature on the relative precision of different predictors which have been reviewed on several occasions (for sheep, Kempster, 1981; for cattle, Kempster, 1983; for sheep, cattle and pigs, Kempster et al. 1982) and only a brief summary is given here. The published results as a whole present a fairly consistent picture for the prediction of carcass lean content indicating that: ( 1 ) body dimensions are poor individual predictors; (2) visual carcass fat assessments, the percentage of perinephric and retroperitoneal 
fat in the carcass, fat thickness and specific-gravity determinations provide greater precision, possibly in that order; (3) sample joints (partial dissections) are the most precise predictors.

When the simpler measurements are combined in multiple regression, their precision approaches that achieved by dissection of a small sample joint. $A$ comparable level of precision is achieved with video-scanning techniques of body cross-sections. For greater precision it is necessary to dissect larger joints or combination of joints but, at high levels of precision, diminishing returns (unit increase in precision/unit increase in costs) set in rapidly (Kempster et al. 1982).

Reference has not been made to visual conformation assessment because this presents a different problem from other predictors, in that variation in fatness has to be removed before a positive relation with carcass lean content is realized. The point is best illustrated for sheep where the value of conformation as a predictor of leanness is very sensitive to the method of adjustment to equal fatness (Kempster et al. 198I). The value of conformation as a predictor in breeding schemes, population studies and experiments is also very dubious, although it does have some value as a breed indicator in classification schemes (Kempster et al. 1982).

The most satisfactory approach to prediction is to construct equations within the particular circumstances (time and sample of carcasses) where they are to be used. But this strategy is rarely practicable except in experiments and population studies. In other applications it is necessary to use prediction relations established elsewhere with the associated risk that results may be biased. I shall return to this problem later. Carcass classification schemes and breeding programmes present quite different prediction problems.

\section{Carcass classification schemes}

The choice of prediction method in carcass classification schemes is usually dominated by the difficulties of measuring carcasses on fast-moving slaughter lines. Classified populations may also show considerable variation in carcass composition differing, as they do, in breed, sex and the rearing systems on which the animals are grown. Under these circumstances an important objective is to minimize bias between different types of carcasses in a population.

Pig carcasses are normally classified using fat-thickness measurements. Fat-thickness measurements taken by probe over the $m$. longissimus in the hind-rib region provide a convenient and relatively precise prediction of carcass-lean content and are becoming the standard measurements in schemes around the world (reviewed by Kempster et al. 1982). However, bias between breed types is a problem. A coordinated trial sponsored by the Commission of the European Communities and involving pig populations in seven countries has demonstrated the extent of the differences that can exist in carcass lean content at the same fat thickness (Commission of the European Communities, 1979). The differences were greatest (five percentage units lean in carcass) between national populations involving blockier breeds of pig including Belgian Pietrain blood and 
those based on White-bred populations. We have also found important differences in carcass lean content at equal fat thickness among pigs from different commercial breeding companies in Britain (Evans \& Kempster, 1979) and have also suggested that relations can change significantly over time (Diestre \& Kempster, 1985).

Reliance on fat-thickness measurements is likely to be called into question increasingly as interest grows in the use of specialist sire lines with higher lean:bone values, and there is a clear need to find measuring techniques related more directly to carcass lean content and less liable to breed bias.

Several instruments are now available which automatically record fat measurements and offer potential advantages over the optical probe in terms of improved data handling, labour savings and reduced operator fatigue. Among these the Hennessy and Chong Grading Probe (Hennessy and Chong Ltd, Auckland, New Zealand) and the SFK Fat-o-Meater (SFK Ltd, Hvidovre, Denmark) are capable of measuring muscle thickness and offer a small improvement in precision over the optical probe currently used to measure fat thickness (Kempster et al. 1985), but are unlikely to remove completely the bias referred to previously. The bias is more closely related to breed differences in conformation and it seems likely that visual conformation assessments will continue to play a role in pig carcass classification for some time to come.

Visual assessments of both external fat cover and conformation are an essential element of 'the state of the art' in beef and sheep carcass classification. The EEC Beef Classification Scheme, which is beginning to have an important effect on marketing policy, is based entirely on such assessments. However, the wording of the regulations of the scheme is a 'committee-table' compromise of rather different national approaches, and some problems of interpretation, as well as standardization, can be expected. The current challenge is to achieve a standard application of the scheme in the different countries.

Bias between breeds in predicted lean content can also be a serious problem for beef and sheep because of breed variation in lean:bone and fat partition. The problems involved and the factors to consider when choosing the most appropriate predictors were discussed by Kempster et al. (1984). An important area for future research is to determine how breed and degree of maturity influence the relations between killing-out percentage, muscling (the thickness of muscles) and muscle:bone value.

Video-based approaches to fat and conformation assessments seem unlikely to provide a cost-effective alternative to visual scores in the immediate future. The principles are sound and the techniques show considerable promise but the difficulties involved in designing sophisticated measuring techniques for routine operation in the harsh abattoir environment should not be underestimated. Experience in the use of automatic probes with pigs indicates that the lag time between research prototypes and large-scale commercial application can be considerable. We have argued that a carcass lean baseline for each class in the EEC Beef Classification Scheme is required to facilitate the use of the new measurement techniques (Kempster et al. 1984). 


\section{Breeding programmes}

The critical factor here is the accuracy with which the body composition of live animals can be estimated because performance testing normally has significant advantages over progeny testing in rate of genetic progress. Breeding populations are much less variable in carcass composition than those evaluated in commercial carcass classification schemes; within the context of a selection index, it may be necessary to determine the differences in body composition between animals of the same live weight for age and total feed consumption.

Ultrasonic techniques have the greatest practical application at present. The equipment available ranges from single machines giving a reading of fat thickness to complex scanning machines capable of producing two-dimensional pictures of cross-sections through the animal body. Fat thickness measurements taken in the rib region by simple A-mode machines like the Meritronics (Meritronics Ltd., Wolverton, UK) can provide a satisfactory level of precision for the prediction of body composition in pigs but cross-sectional scans by more complex machines are normally necessary to provide a comparable level of precision in cattle. Results for sheep are less precise and it is debatable whether ultrasonic techniques can be used effectively in normal sheep-breeding schemes unless fatness variation can be increased (Simm et al. 1985 ).

The position for pigs and beef is very similar, therefore, to that in classification and grading. The ease of measuring fat thickness provides a useful prediction but it is an indirect measurement of leanness restricted to overlying fat in the dorsal parts of the carcass. Again, there is a need for an accurate and direct measurement of leanness.

There is considerable interest around the world in sophisticated techniques for live-body measurement, in particular X-ray measured computer tomography and nuclear magnetic resonance. Recent developments were reviewed in an EEC workshop held at the Agriculture and Food Research Council Meat Research Institute (Lister, 1984). The techniques are highly expensive and although they might find some application in national breeding programmes in the long term, their main application is likely to be in detailed studies of growth and development. Reviewing the cost-effectiveness of techniques at this workshop, I concluded that the requirements of techniques for different applications vary considerably and that there is a major gap between the sophisticated high-cost techniques suitable for use in national breeding schemes and experiments on the one hand, and the more basic requirements for ease of operation in commercial production and marketing applications on the other (Kempster, 1984). The scientific community, in their pursuit of accuracy and consistency, should not forget the operational needs for practicability and efficiency.

There may also be statistical problems associated with the use of complex techniques. If a set of animals is measured in a very sophisticated way, for example by digitizing distributions of $\mathrm{X}$-ray tomograph values at a number of locations down the body and then using partial least-squares techniques to analyse the data 
generated, precision is bound to be improved. But at the same time there will be increasing dependence on any peculiarities or special features in the sample of animals measured and the extra precision may not be realized in other applications. With conventional regression techniques aimed at minimizing variation about the fitted line, the residual standard deviation achieved will be an optimistic measure of the precision likely to be achieved in indirect application of the equation. The question is how optimistic?

Physiological predictors (hormone assays) would have major advantages over most other live-animal evaluation techniques if they could provide an accurate estimate early in life of subsequent growth and carcass composition. The halothane test is an indirect physiological test and the importance now being attached to this technique makes the point well. Continued research to find other methods is, therefore, important.

Carcass evaluation in progeny testing has the same objectives as in populations and experiments, i.e. the comparison of different groups of carcasses (sire progeny groups in progeny testing).

\section{Experiments and population studies}

The most satisfactory approach to prediction in population studies and experiments is to construct equations within the study where they are to be used. Equations can be constructed separately for each treatment or individual treatment intercepts can be used together with pooled within-treatment slopes as in the double-sampling-with-regression procedure (Conniffe \& Moran, 1972; Evans \& Kempster, 1979). The double-sampling technique can be used effectively and anyone considering carcass-evaluation techniques is recommended to explore its use. We have examined the techniques for optimizing the choice of predictors and have developed a simple criterion for doing this (Cook et al. 1983). However, the approaches mentioned previously require some baseline dissection, which is costly to perform, and it is often necessary to use equations developed elsewhere. In such cases, it may be best to use equations developed in circumstances as close as possible to those in the proposed trial. Alternatively, prediction equations known to be stable over a range of circumstances may be used. In neither case is there a guarantee that the estimates of carcass composition will not be biased to some extent.

Although there are many reports of analyses to compare predictors within a single group of carcasses, few workers have examined the same predictors over a series of groups differing in breed type, sex, etc., to determine the stability of prediction equations; sample sizes have generally been too small or the information insufficiently variable in origin to do this. More specific indications have emerged from large-scale breed comparison trials carried out in Britain by the Meat and Livestock Commission (Kempster et al. 1982). Predicting measurements with more stable equations also tend to be those providing more precise prediction which, as indicated earlier, are also the more costly. 
Since no carcass measurements or small sample joints have yet been found which are of outstanding value in relation to cost, such a biological panacea is unlikely to exist and the allocation of valuable resources in haphazard attempts to find it is probably futile. More important is the examination of the underlying features of growth and development in relation to the prediction problem. The kind of questions that need to be asked are as follows: (I) What makes a particular fat thickness measurement good for prediction purposes? Is it its absolute thickness relative to other fat thickness which is important, or its position relative to the structure of underlying muscles, or the rate at which it changes with overall composition? (2) What constitutes a good sample joint? Is it its size, its location in the animal body or the relative proportion of the different tissues which is important? (3) How do treatment, breed and sex differences in tissue growth and development lead to prediction biases?

There are several strategies which can be used to overcome bias when full dissection is not possible. It may be helpful to change predicting measurements between different replicates of experiments or between different generations of breeding programmes. Such a strategy may be particularly important in different generations of breeding programmes since reliance on a single predictor might lead to accumulated biases. There may also be advantages from the use of different sample joints on different carcasses within treatments of the same experiment although the statistics of this approach have not been properly explored. It is also advisable to use a combination of predicting measurements which rely on different carcass characteristics: for example, it would be more desirable to use a combination of fat thickness and specific gravity rather than two fat-thickness measurements (precision being similar).

\section{REFERENCES}

Commission of the European Communities (1979). Development of Uniform Methods of Pig Carcass Classification in the EC. Information on Agriculture Series no. 70. BrusselsLuxembourg: Commission of the European Communities.

Committee on Medical Aspects of Food Policy (1984). Diet and Cardiovascular Disease. Report on Health and Social Subjects no. 28. London: H.M. Stationery Office.

Conniffe, D. \& Moran, M. A. (1972). Biometrics 28, 101 I-1023.

Cook, G. L., Jones, D. W. \& Kempster, A. J. (I983). Animal Production 36, 493-495.

Cross, H. R. (1983). American Meat Science Association, Carcass Composition Guidelines. Proceedings of the 36th Annual Reciprocal Meat Conference, North Dakota State University.

Diestre, A. \& Kempster, A. J. (1985). Animal Production (In the Press) 41.

Evans, D. G. \& Kempster, A. J. (1979). Animal Production 28, 97-108.

Kempster, A. J. (1980). Meat Science 5, 83-98.

Kempster, A. J. (1981). Livestock Production Science 8, 263-271.

Kempster, A. J. (1983). Outlook on Agriculture 12, $147-152$.

Kempster, A. J. (1984). In In Vivo Measurement of Body Composition in Meat Animals, pp. I I-203 [D. Lister, editor]. London and New York : Elsevier.

Kempster, A. J., Chadwick, J. P. \& Jones, D. W. (1985). Animal Production 40, 323-329.

Kempster, A. J., Croston, D. \& Jones, D. W. (I98I). Animal Production 33, 39-49.

Kempster, A. J., Cuthbertson, A. \& Harrington, G. (1982). Carcase Evaluation in Livestock Breeding, Production and Marketing. London: Granada. 
Kempster, A. J., Cuthbertson, A. \& Harrington, G. (1984). In Carcass Evaluation in Beef and Pork: Opportunities and Constraints. Proceedings of the Satellite Symposium to the EAAP, The Hague, 1984, pp. $21-29$ [P. Walstra, editor]. Schoonoord, The Netherlands: IVO.

King, J. W. B. (1981). In In Vivo Estimation of Body Composition in Beef, Report on a Commission of the European Communities Workshop, Copenhagen, December 1981, pp. 86-93 [B. Bech Andersen, editor]. Publication no. 524. Copenhagen: Beretning fra Statens Husdyrbrugs forsøg.

Lister, D. (1984). In Vivo Measurements of Body Composition in Meat Animals. London and New York: Elsevier.

Simm, G., Avalos, E., Dingwall, W. S., King, J. W. B., Pratt, A. C., Owen, M. G. \& Cuthbertson, A. (1985). Selection for improved lean meat production in Suffolk sheep: performance test regime and use of ultrasonics and X-ray computed tomography. British Society of Animal Production Winter Meeting. 